\title{
Relaxation Behavior and Heterogeneous Structures of Metallic Glasses
}

\author{
by
}

\author{
Hiroshi TANIMURA*, Tomoki HAYASHI*, Martin LUCKABAUER ${ }^{* *}$, Tomoya KAWAGUCHI*, \\ Masato WAKEDA, ${ }^{* * *}$ Hidemi KATO* ${ }^{*}$ and Tetsu ICHITSUBO*
}

Key words: Metallic glasses, Complex relaxation, Low temperature properties, EMAR, Heterogeneous structure of glass material

\section{Introduction}

Metallic glasses are amorphous alloys that exhibit a glass transition. Generally, the metallic bond is comparatively weaker than covalent or ionic bonds, so atom rearrangement easily occurs when the melted metal is cooled down, producing a tendency to form crystals with a long-range ordered structure. Therefore, in order to obtain a metal sample with amorphous structure, ultrafast quenching $\left(\sim 10^{5} \mathrm{~K} / \mathrm{s}\right)$ is required in which atom rearrangement does not occur. For this reason, only glass samples with a limited shape (e.g., ribbons, thin films) could be produced in the initial stage of this research field. However, many researches to date have discovered that, by selecting the elements and adjusting the composition, even materials made of metal elements could form an amorphous alloy structure (i.e., so-called metallic glass) even at relatively slow cooling rates $\left(10^{-1}-10^{2} \mathrm{~K} / \mathrm{s}\right){ }^{1}{ }^{1}$ The fact that vitrification is possible even with a slower cooling rate signifies that bulk samples can be obtained. Furthermore, metallic glasses have attracted attention as a new material class due to their superior characteristics not seen in crystalline metal materials used to date, with active research being performed over the past ten years on the materials.

Rapid cooling of the liquid metal hinders atom rearrangement and thus crystallization, resulting in a supercooled liquid state in which atoms can freely move even below the melting point. Further cooling from this point induces a glass transition (i.e., phase transition between supercooled liquid and amorphous solid) at a given temperature, forming an amorphous solid. This glass transition occurs not only when cooling but also when increasing the temperature from the amorphous solid state. Metals that have glass transitions which can be reversibly observed during cooling and heating are called metallic glasses to distinguish them from amorphous metals. Increasing the temperature from the glass transition temperature temporarily leaves the material in a supercooled liquid state, but further increase in temperature induces the re-arrangement of the atoms into stable potential positions due to thermal vibration, inducing crystallization. At this point, the enthalpy difference between the supercooled liquid and crystal is released as crystallization enthalpy. Furthermore, the fact that the glass transition can be observed with increased temperature indicates the extent to which the supercooled liquid state is stable. However, the thermal instability easily induces crystallization even below the glass transition temperature when the temperature was elevated slowly, and our past research showed that rapid increases in temperature can induce the glass transition even for amorphous metals, for which the glass transition was thought to be unobservable. ${ }^{2)}$ As a result, whether the glass transition occurs or not is only an issue of kinetics, and there is no clear standard for distinguishing between metallic glasses and amorphous metals. For convenience, we distinguish metallic glasses, which still become amorphous even when cooled relatively slowly and whose glass transition can be confirmed even when heated relatively slowly (i.e., it is thermally stable) from amorphous alloys.

The glassy state or amorphous state is not a thermodynamic equilibrium state but is instead a metastable state; therefore, it is laborious to consider the thermodynamics of glass materials. The internal structure of metallic glasses has not yet been clarified, with many unanswered questions such as, "why do metallic glasses exist in the metastable glass state regardless of the fact that they are primarily composed of metallic bonds?". This paper briefly describes glass-specific phenomena not seen in crystalline metals (mainly relaxation phenomena) and outlines the low temperature properties of metallic glass, and the relationship with the static elastic heterogeneous structures of glass that we have proposed in recent years.

\section{Glass transition and viscosity}

The glass transition is one of the properties not found in crystalline metals. Here we consider the case that cooling glass from the liquid state at a sufficient rate so as to prevent crystallization. At high-temperature above the melting point, the viscosity of liquids is low, but it increases below the melting point as the temperature decreases. This is because decreasing temperatures reduce the thermal vibration of atoms

\footnotetext{
+ Received Nov. 24, 2020 @ 2021 The Society of Materials Science, Japan

* Institute for Materials Research, Tohoku University, Katahira, Aoba-ku, Sendai, 980-8577 JAPAN

** Faculty of Engineering Technology, University of Twente, Drienerlolaan 5, 7522 NB Enschede, NETHERLANDS

*** National Institute for Materials Science, Sengen, Tsukuba, 305-0047 JAPAN
} 
and make the inter atomic interactions relatively larger. The supercooled liquid is considered to become solid once the shear velocity reaches $10^{13} \mathrm{P}\left(=10^{12} \mathrm{~Pa} \mathrm{~s}\right)$. We define this phenomenon as the glass transition, and the temperature at which this occurs as the glass transition temperature. In silica glass $\left(\mathrm{SiO}_{2}\right)$, an Arrhenius-type relationship is established approximately between viscosity and temperature and it can be expressed as $\eta=\eta_{0} \exp \left(E / k_{B} T\right)$. Here, $\eta$ is the viscosity, $\eta_{0}$ is a constant which represents viscosity at ultra-high temperatures $\left(\sim 10^{-5}\right),{ }^{3)} \quad E$ is the activation energy (constant), $k_{B}$ is Boltzmann's constant, and $T$ is the absolute temperature. However, in many glass-forming liquids, it has been found that the temperature dependence of viscosity deviates from the Arrhenius type; the apparent activation energy changes with temperature as $E \propto d \ln \eta(T) / d(1 / T)$, but the primary causes of such viscous behavior with temperature are not well understood. The relationship between $\eta$ and $T$ is empirically shown using the Vogel-Fulcher-Tammann (VFT) equation: $\eta(T)=\eta_{0} \exp \left[A /\left(T-T_{0}\right)\right]$. Here, $A$ and $T_{0}$ are constants, and the latter is referred to as the VFT temperature, which is the temperature at which the viscosity diverges.

Fragility is the classification of glasses based on these differences in temperature dependence for viscosity. Arrhenius-type materials referred to as strong $\left(T_{0} \approx 0\right)$, and non-Arrhenius-type materials referred to as fragile $\left(T_{0}>0\right)$. Specifically, fragility is expressed using the following equation and determined from the rate of change in viscosity at the glass transition temperature $\left(T_{g}\right)$ as follows:

$$
m=\left.\frac{\partial \log \eta(T)}{\partial\left(T_{g} / T\right)}\right|_{T=T_{g}}
$$

A liquid is considered to be stronger the closer $m$ is to 1 . The $\mathrm{SiO}_{2}$ and $\mathrm{GeO}_{2}$ classified here have a local structure in which $\mathrm{Si}-\mathrm{O}$ and $\mathrm{Ge}-\mathrm{O}$ bonds form a tetrahedral arrangement by the covalent bonds and have extremely strong directionality of the bonds. Conversely, as $m$ becomes larger than 1, a liquid is more fragile; some representative examples include $\mathrm{Pd}_{42.5} \mathrm{Ni}_{7.5} \mathrm{Cu}_{30} \mathrm{P}_{20}(m \sim 50)$, handled in this study.

Furthermore, both types have other characteristics besides temperature dependence of viscosity. For example, strong glasses have a relatively unchanged elastic modulus even when crystallized, whereas fragile glasses can change by up to 30 $50 \%$. Besides that, $\beta$ relaxation, which is a rapid relaxation seen at lower-temperatures (or higher-frequency) than normal glass transitions (as mentioned in Section 3), was clearly observed in fragile glasses, but this phenomenon was difficult to see in strong glasses. $\left.{ }^{4}\right)$

\section{Complex relaxation phenomena}

Various relaxation phenomena such as the glass transition are generally observed in glasses, including metallic glass. A brief explanation is provided here of what relaxation phenomena are, as well as the types and origins of relaxation seen in glass. An equilibrium state shifts into a new equilibrium state by applying external force. These types of phenomena are referred to as relaxation phenomena. ${ }^{5), 6)}$ Since the relaxation generally occurs with the aid of thermal (vibrational) force, the response to an external force is accompanied by a time delay. The time it takes for equilibrium to be reached is referred to as the relaxation time $\tau$, and relaxation phenomena can be expressed in the form of $\exp (-t / \tau)$. Delayed strain responses to stress (displacement occurs because the atom jumps to a new equilibrium position) results in hysteresis in the stress-strain curve, thereby resulting in the loss of energy applied. This energy loss is referred to as internal friction. When the frequency of external force applied to a sample is swept, the peaks of internal friction are observed at specific frequencies. The frequencies at which the peaks occur is determined by the relationship between temperature and the activation energy of relaxation. Thus, relaxation phenomena can be studied from this internal friction measurement.

Next, we refer to the relaxation phenomenon observed in metallic glass. The relaxation which always occurs in metallic glasses is $\alpha$-relaxation, and the phenomenon where this $\alpha$ relaxation is frozen or when the frozen $\alpha$-relaxation can be observed is referred to as the glass transition. Although atoms need to shift to a new equilibrium position for relaxation to occur in $\alpha$-relaxation, this is achieved by cooperative movement of regions of a given size as opposed to single atoms. ${ }^{7)} \alpha$-relaxation is also referred to as "primary relaxation" and can be observed in all metallic glasses (as well as all other glasses). Separately from primary relaxation, there is also "secondary relaxation", known as $\beta$-relaxation. There are systems in which $\beta$-relaxation is easy to observe and others where this relaxation is not easy to observe, with fragile glasses falling in the former case and strong glasses falling into the latter case. ${ }^{8)}$ We believe that this is due to differences in the

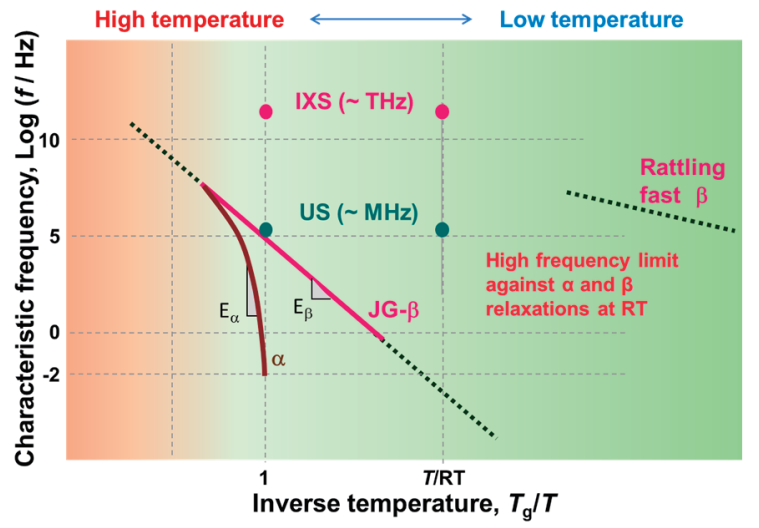

Fig. 1 Schematic figure illustrating the characteristic frequencies of the $\alpha$ and JG- $\beta$ relaxations. The frequency of the relaxation is frozen at $T_{g}$. Both frequencies merge at a high temperature, indicating the fundamental processes of each are the same. 
internal structure of metallic glasses (difference in the degree of static elastic heterogeneity).

The origin of this $\beta$-relaxation is thought to be a local atomic jump, and this can be further divided according to its nature. One is referred to as Johari-Goldstein (JG) $\beta$-relaxation, which is $\beta$-relaxation as an elementary process of $\alpha$-relaxation. ${ }^{9}{ }^{9}$ Both $\alpha$ - and $\beta$-relaxation in this case can be observed to merge at high temperatures above the glass transition temperature, as shown in Fig. 1. This indicates that $\beta$-relaxation, which could only be locally relaxed due to low temperatures, cannot be distinguished from $\alpha$-relaxation when temperatures increase and the atoms move cooperatively. The JG- $\beta$-relaxation is considered to be a local precursor (elementary process) of primary relaxation. The second is behavior that is unrelated to $\alpha$-relaxation and is due to the local rotation of molecular chains found in polymer glass. This non-JG- $\beta$-relaxation is not an elementary process of $\alpha$-relaxation. Metallic glasses are primarily composed of metallic elements and do not have molecular chains, so $\beta$-relaxation that appears in metallic glass is fundamentally JG- $\beta$-relaxation, making it a precursor for $\alpha$ relaxation. There are also cases in glass where boson peaks of relaxation phenomena derived from the random structure of glass and rattling motion (fast $\beta$-relaxation) in frozen cages may be observed in the low-temperature range in addition to $\alpha$ - and $\beta$-relaxation. The low-temperature physical properties found in glass are described below.

\section{Low-temperature properties of glass}

The specific heat $C_{p}$ of a crystalline material can be approximated well with the Debye model, and it is known that this is proportional to $T^{3}$ at extremely low temperatures. The phonon wavelength in the low temperature range is on the order of $0.01-1 \mu \mathrm{m}$, which is sufficiently large compared with the atomic scale. Therefore, the random structure in glass is no longer important in such low temperatures and $C_{p}$ of glass materials had also been considered to follow the Debye cube law. However, it has been revealed that low temperature $C_{p}$ in various metallic glasses deviates significantly from the Debye cube law. ${ }^{10)}$ Anomalies in $C_{p}$ have also been observed in various glasses, and it is considered that they are related to the boson peak. ${ }^{11), 12)}$ The boson peak is a phenomenon in which the vibrational (or phonon) density of states (vDOS) becomes larger than that expected from the Debye model on the lowenergy side and has been observed in glasses, polymers, and non-equilibrium crystals. ${ }^{11), 13)}$ Incidentally, the internal energy is expressed using vDOS, and the first derivative of the internal energy with regard to temperature becomes the specific heat, so increases in vDOS signify an increase in the specific heat. The Debye model assumes a homogeneous and isotropic substance, and the random structure of glass is considered to be the origin of the anomalous boson peak.

From the above reasoning, it is interesting to investigate whether certain atoms can move in the glass structure at such low temperatures to grasp the correlation to the low temperature anomalies. Recently, we carried out the internal friction measurements on $\mathrm{Zr}_{52.5} \mathrm{Cu}_{17.9} \mathrm{Ni}_{14.6} \mathrm{Al}_{10} \mathrm{Ti}_{5}$ (Vit105) after heat treatment at a sub- $T_{\mathrm{g}}$ temperature $\left(T=T_{g}-80 \mathrm{~K}\right)$ for various periods (including as-quenched) by electromagnetic acoustic resonance (EMAR) method. ${ }^{14)}$ Figure 2 shows the temperature dependences of samples, measured in acoustic resonance of the same resonance mode at around $700 \mathrm{kHz}$. We obtained a significantly broad internal friction spectrum regardless of aging time. Thus, large internal friction for this glass material is observed at low temperatures, being associated with the random structure. Especially, it is considered that a "fast $\beta$ relaxation" process greatly contributes to this phenomenon. As shown in the inset of Fig. 2, this process would be caused by a microscopic rattling motion of an atom occurring in the frozen atomic cage structure. Since the activation energy of this process is on the order of several $\mathrm{meV}$, fast $\beta$ relaxation can dominates the large internal friction of glass materials at low temperatures, where other relaxation phenomena such as $\alpha$ relaxation and $\beta$ relaxation are completely frozen, as shown in Fig. 1. On the other hand, the internal friction was largely reduced by aging treatment, i.e., the progress of structural relaxation. Especially, it deserves to note that a large reduction of internal friction was observed even in the short time heat treatment of 60 seconds. This result clarified that the local structural relaxation which changes the macroscopic physical properties can progress even in the short time heat treatment at temperature sufficiently lower than $T_{g}$.

This cage structure would be the microscopic origin of free volume of glass materials. So far, low temperature internal friction of glasses has been studied mainly for silica glass. ${ }^{15)}$ In these studies, the fast $\beta$ relaxation was approximated as the vibrational motion of atoms in asymmetric double-well

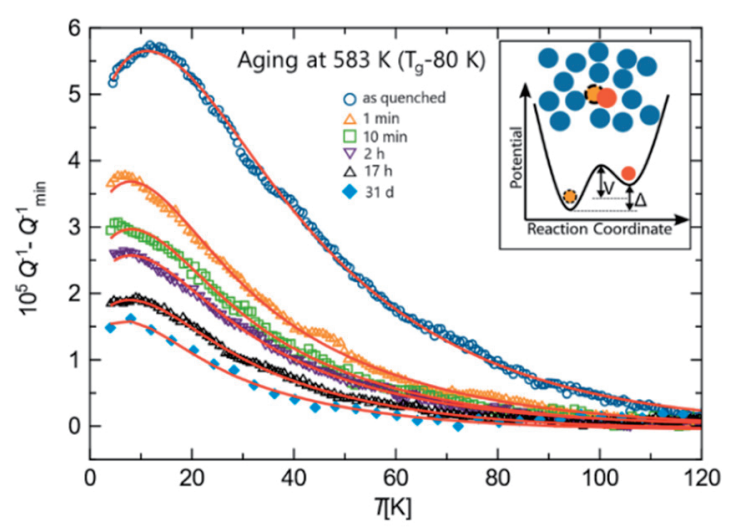

Fig. 2 Temperature dependence of internal friction of Vit105, heattreated for different time durations at $583 \mathrm{~K}$, which was measured at around $700 \mathrm{kHz}{ }^{14}$ ) The inset schematically shows fast $\beta$ relaxation in the atomic cage of glass material. This process can be approximated by atomic vibrations in asymmetric double well potentials. In the TLS model, this potential is characterized by activation energy $V$ and asymmetry $\Delta$. 


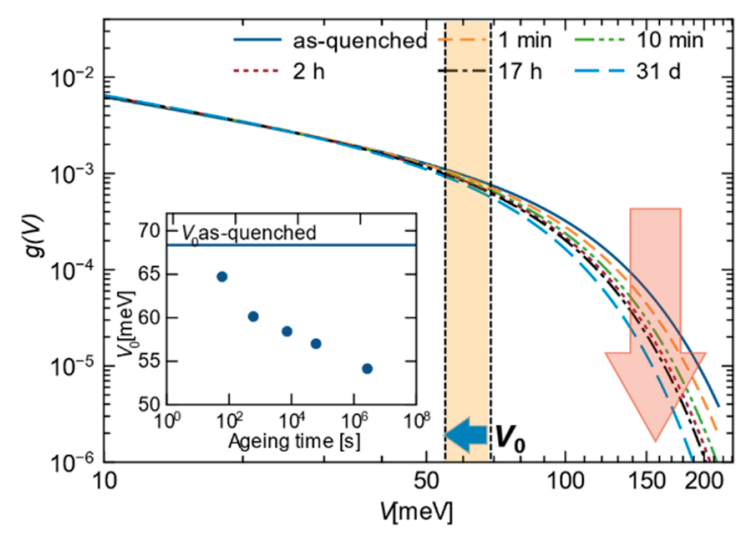

Fig.3 Energy distribution function $\mathrm{g}(\mathrm{V})$ of heat-treated Vit105 calculated based on TLS model. ${ }^{14)}$ Inset: Decrease in fitting parameter $\mathrm{V}_{0}$ due to structural relaxation. This means that TLS with large activation energies is preferentially reduced.

potentials (ADWPs, inset in Fig. 2). Moreover, when the distance between metastable positions in ADWP is sufficiently short, it is possible to simplify ADWP as a two-level system (TLS), which is characterized by only 2 parameters of activation energy $V$ and asymmetry $\Delta$. The analysis based on the TLS model successfully explained low temperature internal friction of the silica glass. In the same way, we investigated the correlation between structural relaxation and fast $\beta$ relaxation of metallic glass, by adopting above TLS model for the analysis of the low temperature internal friction measurement. The detailed analyses are shown in the literature. ${ }^{14)}$ The solid lines in Fig. 2 show the analysis results based on the TLS model. The observed temperature dependences of internal friction are well reproduced, indicating that the TLS model which has been developed for silica glass can also be used for metallic glass.

In order to analyze the effect of structural relaxation with TLS in more detail, the distribution function of TLS, $g(V)$, was calculated by a theoretical formula; the results are shown in Fig. 3. In this study the distribution function of TLS, which is originally described as two-variable function $\left.P(\Delta, V),{ }^{15}\right)$ was simplified as $P(\Delta, V)=g(V) f(\Delta)$ by assuming $V$ and $\Delta$ are independent of each other. It was clarified that the distribution of TLS with larger activation energy decreased preferentially with the progress of structural relaxation. This result indicates that, in the actual glass structure, the volume of the cage structure wherein fast $\beta$ relaxation occurs decreases preferentially, i.e., that the ADWPs approach to single-well potential (toward an "ideal glass" structure). This shrinkage of the cage structure can be regarded as the microscopic origin of the reduction of free volume of metallic glasses by heat treatment.

\section{Structural model of metallic glass}

We have conducted various studies on the physical properties and structure of metallic glasses. It is thought from these results that metallic glasses have static heterogeneous structures, and that this may play an important role in various physical properties such as fragility and relaxation behavior. The static heterogeneous structure model of the internal structure of metallic glasses is described in this section.

\section{$5 \cdot 1$ Heterogeneous structure seen in $\mathrm{Pd}_{42.5} \mathrm{Ni}_{7.5} \mathrm{Cu}_{30} \mathrm{P}_{20}$}

The research that we have conducted on the crystallization behavior of the fragile metallic glass $\mathrm{Pd}_{42.5} \mathrm{Ni}_{7.5} \mathrm{Cu}_{30} \mathrm{P}_{20}$ (hereinafter referred to as PNCP) and the structural model estimated from these results are described. ${ }^{4)}$ Enhancement of crystallization occurred when PNCP was maintained at an isothermal temperature near/below the glass transition temperature (approximately $50-90 \quad \mathrm{~K}$ lower than the crystallization temperature) while ultrasonic vibration of several MHz was applied using the EMAR method. The observed behavior was different from that of normal crystallization. A high-resolution electron microscopy (HREM) image taken after the experiment and a structural model estimated from this are shown in Fig. 4. Crystal grains about a micrometer in size are usually precipitated independently from the amorphous phase in the crystallization process in this system, but as seen in Fig. 4 it can be observed that the crystal phase is formed so as to surround the amorphous phase in this case. This mechanism of crystallization can be thought of as follows.

An irreversible atomic jump is induced as a result of the resonance of the atomic motion relating to $\beta$-relaxation (its frequency comparable to the present ultrasound frequency) and the potential modulations caused by ultrasonic vibration. It is thought that repeated irreversible atomic jumps eventually lead to the potential position of the crystal being reached, which in turn advances crystallization regardless of the fact that the temperature is much lower than the crystallization temperature. The potential is easily modulated by ultrasonic waves, and it is estimated that the section where crystallization was induced by constant temperature aging under ultrasonic vibration is the weakly bonded region (low-shear modulus region), while the section where the amorphous phase is maintained is thought to be the strongly bonded region. From there, the structural model of PNCP was proposed. ${ }^{4)}$ This model suggests that the strongly bonded region (SBR) surrounds the weakly bonded region (WBR) since the crystalline phase surrounds the amorphous
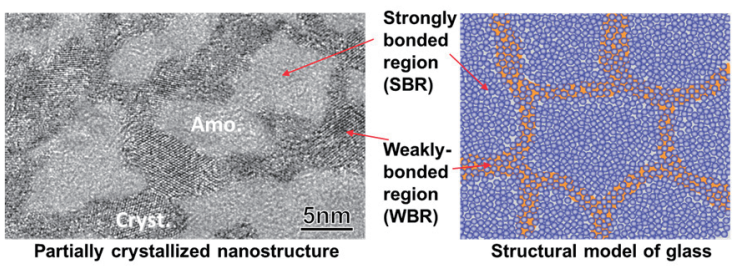

Fig. 4 (Left) HREM image of partially crystallized PNCP obtained by annealing just below $T_{g}$ under ultrasonic perturbations of submegahertz frequency; amorphous region is colored gray for conspicuity. ${ }^{4)}$ (Right) The inferred inhomogeneous structure model of metallic glass, consisting of strongly bonded regions (SBR) surrounded by weakly bonded region (WBR). 
phase. This structural model has been shown to successfully reproduce the characteristics of fragile glass, such as a large increase in elastic modulus during crystallization and a large Poisson's ratio. Furthermore, this type of elastic heterogeneous structure is distinguished from dynamic heterogeneity and referred to as static elastic heterogeneity. It is also interesting that molecular dynamics simulations suggest a similar structure in single-atom glasses.

\section{5 - 2 Inelastic X-ray scattering study}

The above-mentioned structural model was proposed for PNCP, but is this applicable for other fragile glasses? Furthermore, what is the structure of strong glasses like? These questions remain unanswered. A more accurate understanding can also be obtained by investigating the heterogeneous structure of amorphous metals without inducing crystallization, since the original structure is considered from the structure in which partial crystallization has occurred. Therefore, we studied the microstructure of metallic glass using a technique called inelastic X-ray scattering (IXS). ${ }^{16)-18)}$

IXS is inelastic scattering using X-rays, with which the exchange of energy between X-rays and substances can be observed. The energy exchanged in this process is the excitation energy of phonons, and IXS can obtain information on the structure of matter on the order of $\mathrm{nm}$, since the wavelength of excited phonons is on the order of nm. Fig. 5 shows the phonon dispersion relationships and sound velocity ( $\left.v_{\text {IXS }}\right)$ of six types of metallic glasses obtained from IXS experiments conducted at BL35XU in SPring-8: $\mathrm{Pd}_{42.5} \mathrm{Ni}_{7.5} \mathrm{Cu}_{30} \mathrm{P}_{20}$ (PNCP), $\mathrm{Pd}_{40} \mathrm{Ni}_{40} \mathrm{P}_{20}$ (PNP), $\mathrm{Pd}_{46} \mathrm{Cu}_{35.5} \mathrm{P}_{18.5}$ (PdCP), $\mathrm{Zr}_{50} \mathrm{Cu}_{40} \mathrm{Al}_{10}$ (ZCA), $\mathrm{Zr}_{65} \mathrm{Al}_{7.5} \mathrm{Ni}_{10} \mathrm{Cu}_{17.5}$ (ZANC), and $\mathrm{Zr}_{65} \mathrm{Al}_{7.5} \mathrm{Ni}_{10} \mathrm{Pd}_{17.5}$ (ZANP). The dashed line shown in the figure is the sound velocity ( $\left.v_{U S}\right)$ determined using ultrasonic wave (specifically resonant ultrasound spectroscopy (RUS)). A sound velocity of a wave whose wavelength is on the order of $\mathrm{mm}$ is determined from the ultrasonic wave, and the ultrasound velocity can be regarded as the long wavelength limit. The sound velocity obtained from IXS should normally not exceed this since the sound velocity is at a maximum at the long wavelength limit. However, it can be seen in some types of metallic glass that the sound velocity obtained from the IXS experiments exceeds $v_{U S}$ in the low-Q region. This behavior is schematically shown in the inset in Fig. 5.

The relationship $v=C / \rho$ ( $\rho$ : density) holds between the elastic constant $C$ and sound velocity $v$ of the sample, so the difference in sound velocity can reflect the difference in the elastic constant. The sound velocity determined from the ultrasonic wave is the sound velocity of a wave on the order of $\mathrm{mm}$, and it is thought to reflect the macroscale elastic modulus of the entire substance. Meanwhile, vIXs is the sound velocity on the order of nm, and it is thought that there is a part within the sample on the order of $\mathrm{nm}$ which is harder than the

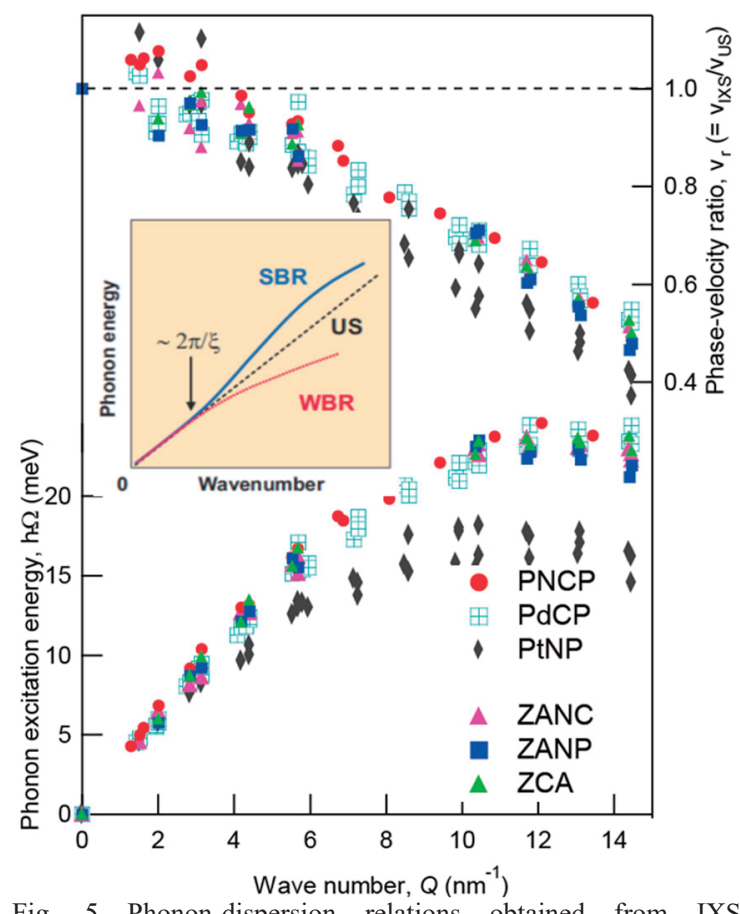

Fig. 5 Phonon-dispersion relations obtained from IXS the phonon-dispersion relation of PNCP. The sound velocity is the phase velocity evaluated from the dispersion relation and $v$ US is the ultrasound speed obtained from the RUS technique. ${ }^{17)}$

macroscale elastic modulus, due to the fact that this sound velocity exceeds the macroscale sound velocity. The IXS experimental results can be successfully explained with the $\mathrm{SBR} / \mathrm{WBR}$ model, assuming that this part which is harder than the macroscopic elastic modulus corresponds to the SBR mentioned earlier. To reiterate, it was stated in Section $\mathbf{5} \cdot \mathbf{1}$ that there are strongly bonded regions (SBRs) surrounded by weakly bonded regions (WBRs) in the PNCP. The SBRs are elastically hard regions, so waves with a wavelength similar to that of the size of the SBRs exhibit a fast sound velocity (=

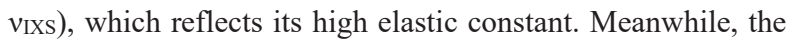
overall elastic constant is the average value of the SBRs and WBRs, and the sound velocity (= $\left.v_{\mathrm{US}}\right)$ obtained becomes slower. As a result, it is thought that $v_{\mathrm{IXS}}>v_{\mathrm{US}}$ in the low-Q region. Furthermore, the size of the SBRs in PNCP were estimated to be $\sim 4-5 \mathrm{~nm}$ given that the phonon propagation distance sharply increased from a wavelength of approximately 4-5 nm. ${ }^{17)}$ From these results, the SBR/WBR structural model estimated from the ultrasonic-wave-promoted partial crystallization experiment were considered valid from the IXS results.

Below, the results of IXS measurements on various metallic glasses other than PNCP with different fragility values are described. A phonon dispersion relationship similar to that of PNCP was determined, and heterogeneity was considered after determining it from this relationship as well as from ultrasound experiments. ${ }^{18)}$ The relationship between $\gamma_{\max }=\left(v_{\mathrm{IXS}} / v_{\mathrm{USS}}\right)^{2} \max$ and fragility is shown in Fig. 6 . The $\gamma$ max value is related to 


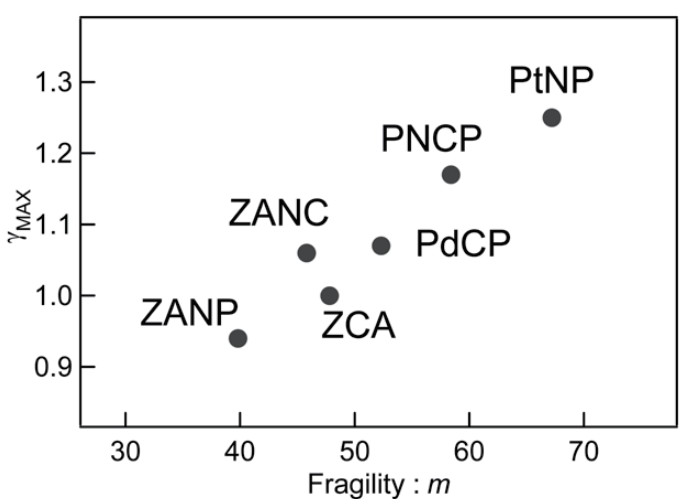

Fig. 6 Relation between the elastic inhomogeneity $\gamma_{\text {MAX }}$ and fragility $\mathrm{m}$ in the six kinds of metallic glasses studied. ${ }^{18)}$

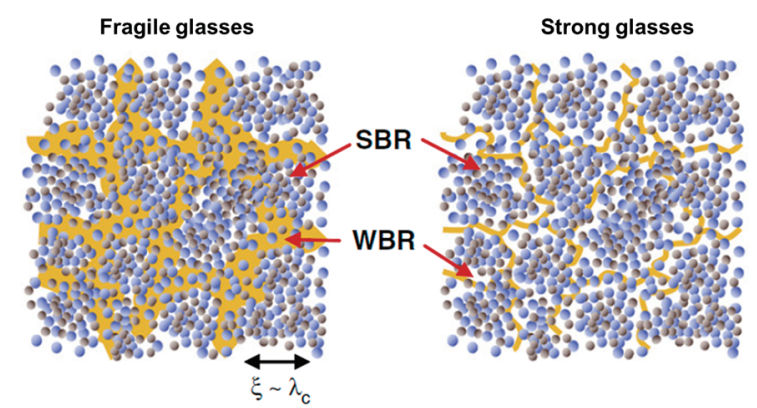

Fig. 7 The inhomogeneous structure models for fragile glass (left) and strong glass (right). Both models consist of strongly bonded regions (SBRs) and weakly bonded regions (WBRs), but the degree of inhomogeneity would be dominated by the fraction of each. ${ }^{18)}$

the increase in the elastic modulus of SBR, compared to the macroscale elastic modulus based on the $C \approx \rho v^{2}$ relationship, and expresses the degree of elastic heterogeneity. It can be seen from Fig. 6 that metallic glasses with higher fragility have higher elastic heterogeneity, and those that are stronger have lower elastic heterogeneity. These results suggest that a structural model on fragile and strong glasses in Fig. 7 is reasonable, which has recently been rationalized. ${ }^{19)}$ In other words, fragile glass has a relatively large volume-fraction of WBRs and high heterogeneity, whereas strong glass has a low volume-fraction of WBRs and a relatively homogeneous and densely packed structure. In addition, our recent work using molecular dynamic simulations will prove that the static heterogeneity is dispersed heterogeneously according as the fragility of metallic glasses.

\section{Conclusions}

Metallic glasses can stably be formed/fabricated in a metastable/nonequilibrium amorphous state, despite being primarily composed of metallic bonding with low bondanisotropy. Currently, active research is being conducted on the origins of this stability and its internal structure. In this paper, we described our recent results on the fast $\beta$ relaxation process and heterogeneity of metallic glasses. The phonon dispersion relationships of six metallic glasses were obtained from inelastic scattering using high-intensity X-rays at BL35XU in
SPring-8. From here, the sound velocity of waves with a wavelength on the order of $\mathrm{nm}$ was determined and resonant ultrasound spectroscopy was used to determine the sound velocity of waves with a wavelength on the order of nm. From these ultrasonic attenuation methods, sound velocity measurements and experiments/computational simulations which used the wavelength-dependency of nanometerwavelength sound velocity and propagation distance, it was suggested that metallic glasses have a static elastic heterogeneous structure comprising of regions with strong bonds and densely packed atoms as well as sparsely packed regions with weak bonding strength.

One of the authors, T.I. (tichi@imr.tohoku.ac.jp), is very gratitude to Emeritus Professor Eiichiro Matsubara (Kyoto University) and Mr. Shunsuke Yukitani (who was a student at Kyoto University). Owing to their great support, T.I. could conduct the metallic glass study project. In carrying out the inelastic X-ray scattering (IXS) experiment, we would like to express our deep gratitude to Dr. A.Q.R. Baron and Dr. S. Tsutsui in this study RIKEN SPing-8/BL35XU and to Professor S. Hosokawa of Kumamoto University for their great cooperation.

\section{References}

1) A. Inoue, "Stabilization of metallic supercooled liquid and bulk amorphous alloys", Acta Materialia, Vol.48, No.1, pp.279-306 (2000).

2) T. Ichitsubo, E. Matsubara, H. Numakura, K. Tanaka, N. Nishiyama and R. Tarumi, "Glass-liquid transition in a less-stable metallic glass", Physical Review B, Vol.72, 052201 (2005).

3) H. Kato, T. Wada, M. Hasegawa, J. Saida, A. Inoue and H. S. Chen, "Fragility and thermal stability of Pt- and Pdbased bulk glass forming liquids and their correlation with deformability" Scripta Materialia 54 (2006) 20232027.

4) T. Ichitsubo, E. Matsubara, T. Yamamoto, H. S. Chen, N. Nishiyama, J. Saida and K. Anazawa, "Microstructure of fragile metallic glasses inferred from ultrasoundaccelerated crystallization in Pd-based metallic glasses", Physical Review Letters, Vol.95, 245501 (2005).

5) Y. Hiki, "Dansei hidansei", Kyoritsu Shuppan.

6) Y. Wada and A. Ikushima, "Choonpa spectrscopy kisohen", Baifukan.

7) G. Adam and J. H. Gibbs, "On the temperature dependence of cooperative relaxation properties in glassforming liquids", Journal of Chemical Physics, Vol.43, pp.139-146 (1965).

8) Z. F. Zhao, P. Wen, C. H. Shek and W. H. Wang, "Measurements of slow $\beta$-relaxations in metallic glasses and supercooled liquids", Physical Review B, Vol.75, 174201 (2007).

9) K. L. Ngai and M. Paluch, "Classification of secondary 
relaxation in glass-formers based on dynamic properties", Journal of Chemical Physics, Vol.120, pp.857-873 (2004).

10) Y. Li, P. Yu and H. Y. Bai, "Study on the boson peak in bulk metallic glasses", Journal of Applied Physics, Vol.104, 013520 (2008).

11) L. Orsingher, A. Fontana, E. Gilioli, G. Carini, Jr., G. Carini, G. Tripodo, T. Unruh and U. Buchenau, "Vibrational dynamics of permanently densified $\mathrm{GeO} 2$ glasses: densification-induced changes in the boson peak", Journal of Chemical Physics, Vol.132, 124508 (2010)

12) Y. Li, H. Y. Bai, W. H. Wang and K. Samwer, "Lowtemper-ature specific-heat anomalies associated with the boson peak in CuZr-based bulk metallic glasses", Physical Review B, Vol.74, 052201 (2006).

13) R. P. Hermann, R. Jin, W. Schweika, F. Grandjen, D. Mandrus, B. C. Sales and G. J. Long, "Einstein Oscillators in Thallium Filled Antimony Skutterudites", Physical Review Letters, Vol.90, 135505 (2003).

14) M. Luckabauer, T. Hayashi, H. Kato and T. Ichitsubo, "Decreasing activation energy of fast relaxation processes in a metallic glass during aging", Physical Review B, Vol.99, 140202 (R) (2019).

15) R. Vacher, E. Courtens and M. Foret, "Anharmonic versus relaxational sound damping in glasses. II. Vitreous silica", Physical Review B, Vol.72, 214205 (2005).

16) T. Ichitsubo, S. Hosokawa, K. Matsuda, E. Matsubara, N. Nishiyama, S. Tsutsui and A. Q. R. Baron, "Nanoscale elastic inhomogeneity of a Pd-based metallic glass: Sound velocity from ultrasonic and inelastic $\mathrm{x}$-ray scattering experiments", Physical Review B, Vol.76, 140201 (R) (2007).

17) T. Ichitsubo, W. Itaka, E. Matsubara, H. Kato, S. Biwa, S. Hosokawa, K. Matsuda, J. Saida, O. Haruyama, Y. Yokoyama, H. Uchiyama and A. Q. R. Baron, "Elastic inhomogeneity and acoustic phonons in Pd-, Pt-, and $\mathrm{Zr}$ based metallic glasses", Physical Review B, Vol.81, 172201 (2010).

18) T. Ichitsubo, H. Kato E. Matsubara, S. Biwa, S. Hosokawa, K. Matsuda, H. Uchiyama and A. Q. R. Baron, "Static heterogeneity in metallic glasses and its correlation to physical properties", Journal of NonCrystalline Solids, Vol.357, pp.494-500 (2011).

19) S. V. Ketov, Y. H. Sun, S. Nachum, Z. Lu, A. Checchi, A. R. Beraldin, H. Y. Bai, W. H. Wang, D. V. LouzguineLuzgin, M. A. Carpenter \& A. L. Greer, "Rejuvenation of metallic glasses by non-affine thermal strain", Nature 524, 200 (2015) 